\title{
Comportamiento de la vitamina $C$ en un \\ producto a base de lactosuero y pulpa de mango \\ variedad Magdalena River (Mangífera Indica I.) \\ durante el secado por aspersión
}

\author{
Behavior of vitamin $\mathrm{C}$ in a product \\ based on whey and variety Magdalena \\ River mango (Mangifera Indica I.) \\ during spray drying
}

\begin{abstract}
The mango of hilacha and whey are two perishables with a lot of nutrients. The objective of this research was to evaluate the conditions of spray drying of a product based on these raw materials trying to get the maximum recovery of vitamin $C$. The product was dried at inlet temperatures of hot air between $120^{\circ} \mathrm{C}$ - $160^{\circ} \mathrm{C}$ and outlet $65^{\circ} \mathrm{C}$ to $74^{\circ} \mathrm{C}$ using maltodextrin between $15 \%-35 \%$. The concentration of vitamin C (AOAC 967.21/90) and other parameters such as hygroscopicity, solubility and color of the product was evaluated. The best drying conditions were defined using a concentration of $27,5 \%$ maltodextrin, inlet and outlet temperatures of the air of $138,2^{\circ} \mathrm{C}$ and $69,6^{\circ} \mathrm{C}$ with a speed of $24908 \mathrm{rpm}$ spraying, obtaining a recovery of vitamin $C$ $15,60 \mathrm{mg} / 100 \mathrm{~g}$ of product $(58,15 \%)$ and over $80 \%$ of the other constituents studied.

Key words: ascorbic acid, maltodextrina, spray drying.
\end{abstract}

\section{INTRODUCCIÓN}

Hoy en día se elaboran gran cantidad de productos a base de lactosuero (1-3), cuya composición nutricional depende la calidad y tipo de suero empleado (dulce o ácido), clase y proporción de materias primas utilizadas para la elaboración del producto y tipo de proceso a que sean sometidas (fermentación, pasteurización, refrigeración).

El lactosuero es definido como el subproducto líquido obtenido tras la precipitación y separación de la caseína de la leche durante la elaboración del queso y constituye aproximadamente $85 \%$ a $90 \%$ del volumen de la leche, cuyos componentes principales son el calcio, la lactosa, sales minerales y proteínas (4-6). Entre las proteínas que se encuentran en el lactosuero tenemos en mayor proporción la $\beta$-Lactoglobulina y $\alpha$-Lactoalbúmina, además de lactoferrina, lactoperoxidasa, inmunoglobulinas y glicomacropéptidos, las cuales desempeñan un importante papel nutritivo como fuente balanceada de aminoácidos esenciales (7-10).
Fernando A. Mendoza-Corvis $(1,2)$ Margarita Arteaga M. (1)

Omar Pérez S. (1)

(1) Programa de Ingeniería de Alimentos. Facultad de Ingeniería. Universidad de Córdoba. Montería - Colombia. (2) Facultad de Ingeniería. Universidad de Sucre. Sincelejo - Colombia

Dirigir la correspondencia a: Profesor

Fernando Mendoza Corvis Programa de Ingeniería de Alimentos

Facultad de Ingeniería Universidad de Córdoba Colombia

Teléfono: 3128788627

E-mail:fmendoza@correo.unicordoba.edu.co

Este trabajo fue recibido el 12 de Septiembre de 2015 y aceptado para ser publicado el 1 de Febrero de 2016.

El mango (Mangífera indica) es originario del sur de Asia y es una de las más conocidas frutas tropicales, con más de 500 variedades (11). Su área de producción en Colombia el año 2014 ascendió a 24.000 hectáreas, con una producción de 280.000 toneladas, la cual en su mayoría se comercializó como producto fresco y del total de la producción nacional, $39 \%$ corresponde a la variedad del mango de hilacha. El mango además de ser un alimento, es fuente de compuestos bioactivos como ácido ascórbico y dehidroascórbico, $\beta$-carotenos, polifenoles, fibra, terpenoides y antioxidantes minerales (12). La vitamina $C$ es reconocida por su participación en el buen funcionamiento del sistema inmune, por poseer actividad antiescorbuto, favorecimiento de la absorción intestinal de hierro no hémico, metabolización de la grasa y como agente antioxidante, por lo que los alimentos que la contiene son considerados de mayor calidad tecnológica y valor nutricional (13), sin embargo se caracteriza por ser una de las vitaminas más termosensibles durante los tratamientos térmicos, razón 
por la cual es usada como indicador de degradación térmica de nutrientes durante el procesamiento de los alimentos.

Mediante esta investigación se buscó aprovechar el uso de materias primas ricas en nutrientes, con poco valor agregado en nuestra región y que son altamente perecederas, en la elaboración de un producto en polvo de mayor accesibilidad y disponibilidad que conservara sus constituyentes. Para ello se utilizó la técnica de secado por aspersión, la cual trabaja a elevadas temperaturas, por lo que se evaluó el efecto de las condiciones de proceso, entre ellas las temperaturas de entrada y salida del aire caliente, la velocidad de aspersión y la proporción de agente encapsulante termoprotector (maltodextrina), en la concentración en vitamina $C$ de un producto elaborado a partir de mango de hilacha (Mangífera indica L.) y lactosuero, ya que este en uno de los constituyentes mas termosensibles, además de otras propiedades como la solubilidad, higroscopicidad y color del producto, buscando lograr la máxima recuperación de los demás nutrientes.

\section{MATERIALES Y MÉTODOS}

Preparación de los tratamientos

La formulación base a secar se preparó con lactosuero de vaca (69\%), pulpa de mango de hilacha (Mangífera indica L.) (24\%) y azúcar (7\%), la cual fue procesada empleando un secador por atomización marca Vibrasec modelo PASALAB 1.5, controlando la temperatura de entrada del aire caliente entre $120^{\circ} \mathrm{C}-160^{\circ} \mathrm{C}$, temperatura del aire de salida entre $65^{\circ} \mathrm{C}-$ $74^{\circ} \mathrm{C}$, velocidad de aspersión entre 20.000 - 28.000 rpm y una concentración de maltodextrina como agente encapsulante entre 15\% a 35\%. Empleando un diseño central compuesto se Ilevaron a cabo 22 tratamientos con 6 repeticiones del punto central y 3 de los puntos axiales (tabla 1). Todos los análisis de realizaron por triplicado.

\section{Determinación de la vitamina $C$}

Se empleó el método de la AOAC 967.21/90. Para ello se preparó una solución de 2,6-diclorofenolindofenol a 400 ppm la cual se utilizó para titular aproximadamente 5 g de cada una de las muestras, que habían sido previamente diluidas en $20 \mathrm{~mL}$ de ácido oxálico $2 \%$, hasta obtener un color rosa constante. Se tituló además 0,2 $\mathrm{mL}$ de una solución patrón de ácido ascórbico 0,2 \% (p/v) y 0,2 mL de agua destilada empleada como blanco. Los resultados se expresaron en $\mathrm{mg}$ de vitamina $C$ por $100 \mathrm{~g}$ de muestra y en porcentaje de recuperación de vitamina $C$.

\section{Solubilidad}

La solubilidad se determinó por el método descrito por Eastman y Moore (1984) (14), modificado por Cano Et al. (2005) (15). Se pesó $1 \mathrm{~g}$ de muestra y se adicionaron a 100 $\mathrm{mL}$ de agua destilada, agitando a alta velocidad durante 5 minutos. La solución se colocó en tubos de ensayo y se centrifugaron a $3000 \mathrm{rpm}$ durante 5 minutos. Una alícuota de $25 \mathrm{~mL}$ del sobrenadante se transfirió a crisoles, previamente

\section{TABLA 1}

Concentración de vitamina C (mg/100 g) en el producto en polvo obtenido.

\begin{tabular}{|c|c|c|c|c|c|}
\hline Tratamiento & $\begin{array}{c}(\mathrm{A}) \\
\text { Maltodextrina } \\
\%\end{array}$ & $\begin{array}{l}(\mathrm{B}) \\
\text { T.E. } \\
\left({ }^{\circ} \mathrm{C}\right)\end{array}$ & $\begin{array}{l}\text { (C) } \\
\text { V. A. } \\
\text { (rpm) }\end{array}$ & $\begin{array}{c}\text { (D) } \\
\text { T.S. } \\
\text { T.S. }\left({ }^{\circ} \mathrm{C}\right)\end{array}$ & $\begin{array}{c}\text { Vitamina } C \text { en el } \\
\text { producto en polvo } \\
\mathrm{mg} / 100 \mathrm{~g}\end{array}$ \\
\hline 1 & 25 & 160 & 24000 & 71 & $2,71 \pm 0,02$ \\
\hline 2 & 20 & 150 & 22000 & 74 & $9,64 \pm 0,11$ \\
\hline 3 & 25 & 140 & 24000 & 77 & $7,38 \pm 0,01$ \\
\hline 4 & 25 & 140 & 20000 & 71 & $11,35 \pm 0,03$ \\
\hline 5 & 35 & 140 & 24000 & 71 & $2,70 \pm 0,001$ \\
\hline 6 & 30 & 150 & 26000 & 68 & $4,00 \pm 0,005$ \\
\hline 7 & 25 & 140 & 24000 & 71 & $22,55 \pm 0,09$ \\
\hline 8 & 25 & 120 & 24000 & 71 & $4,35 \pm 0,01$ \\
\hline 9 & 25 & 140 & 24000 & 65 & $11,09 \pm 0,04$ \\
\hline 10 & 30 & 130 & 26000 & 74 & $4,54 \pm 0,02$ \\
\hline 11 & 25 & 140 & 28000 & 71 & $12,48 \pm 0,08$ \\
\hline 12 & 25 & 140 & 24000 & 71 & $21,60 \pm 0,15$ \\
\hline 13 & 25 & 140 & 24000 & 71 & $18,88 \pm 0,09$ \\
\hline 14 & 20 & 130 & 26000 & 68 & $8,76 \pm 0,03$ \\
\hline 15 & 30 & 150 & 22000 & 68 & $4,28 \pm 0,16$ \\
\hline 16 & 20 & 130 & 22000 & 68 & $9,33 \pm 0,18$ \\
\hline 17 & 30 & 130 & 22000 & 74 & $4,90 \pm 0,08$ \\
\hline 18 & 20 & 150 & 26000 & 74 & $9,87 \pm 0,20$ \\
\hline 19 & 25 & 140 & 24000 & 71 & $14,76 \pm 0,84$ \\
\hline 20 & 25 & 140 & 24000 & 71 & $15,07 \pm 0,62$ \\
\hline 21 & 15 & 140 & 24000 & 71 & $10,22 \pm 0,06$ \\
\hline 22 & 25 & 140 & 24000 & 71 & $22,45 \pm 0,75$ \\
\hline
\end{tabular}

T. E.: temperatura de entrada. V. A.: velocidad de aspersión. T. S.: temperatura de salida. 
secados y tarados, secándose en estufa a $105^{\circ} \mathrm{C}$ por 24 horas. El porcentaje de solubilidad se calculó por diferencia de peso.

Evaluación de la higroscopicidad

Para la determinación de la higroscopicidad se empleó el método descrito por Caí y Corke (2000) (16). Para ello se pesó aproximadamente $1 \mathrm{~g}$ de cada muestra de polvo en cajas de Petri, colocándose a $25^{\circ} \mathrm{C}$ en un recipiente hermético de vidrio lleno de solución saturada de cloruro de sodio. Las muestras se pesaron durante una semana y se expresó como gramos de humedad por $100 \mathrm{~g}$ de sólidos.

\section{Evaluación del color}

La medición del color $\left(L^{*}, a^{*}, b^{*}\right)$ se llevó a cabo en un Colorímetro Color Flex EZ marca HunterLab utilizando un ángulo de observación de $10^{\circ}$ y un iluminante estándar D65. Mejores condiciones de secado

Se determinaran empleando el software Desing expert 8.0.6 buscado obtener el mayor porcentaje de recuperación de

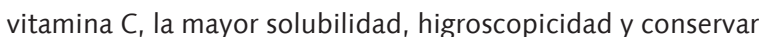
el color del producto.

Análisis de datos

Los datos obtenidos de los tratamientos se analizaron empleando el software Desing expert 8.0.6 con un nivel de significancia del 5\%.

\section{RESULTADOS Y DISCUSIÓN \\ Vitamina $C$}

La concentración de la vitamina $C$ y el porcentaje de recuperación en el producto en polvo obtenido se muestran en la tabla 1 y en la figura 1. Este parámetro presentó diferencia significativa $(p<0,05)$ para los factores $A$ (maltodextrina), D (temperatura de salida), las interacciones lineales $A B$ (maltodextrina - temperatura de entrada), AD (maltodextrina temperatura de salida) y las interacciones cuadráticas A2, B2, C2, D2, (tabla 2) cuyos efectos se denotan en los puntos de inflexión ubicados cerca de los puntos centrales de los trata-

FIGURA 1

Recuperación de la vitamina $C$, solubilidad e higroscopicidad en el producto en polvo.
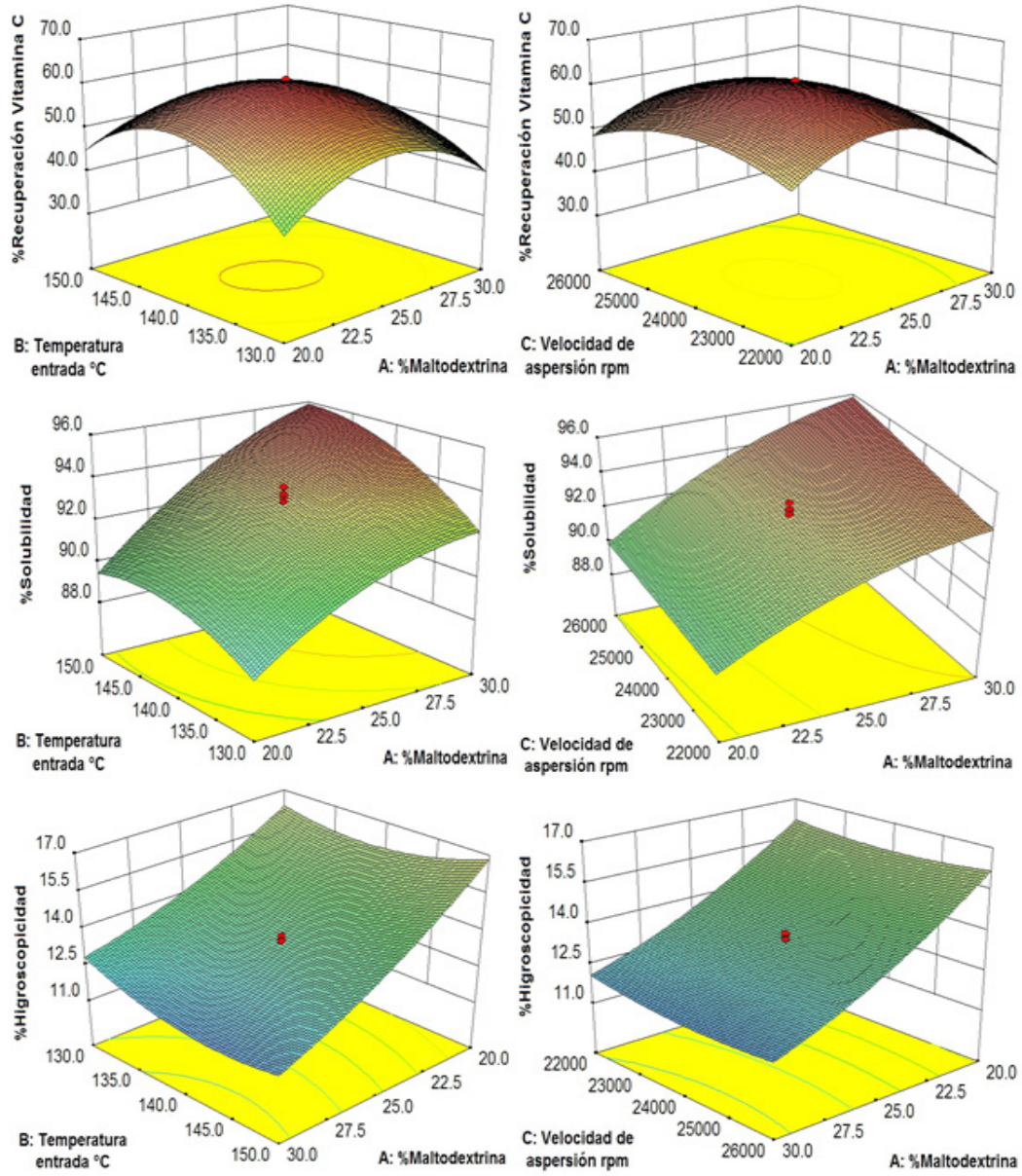
mientos realizados. El porcentaje de recuperación de vitamina $C$ estuvo influenciado por la concentración de maltodextrina (MD) en la formulación, observándose inicialmente su incremento al pasar de $54,19 \%$ con $20 \%$ de MD a $65,17 \%$ con $25 \%$ de $M D$, esto debido probablemente al aumento de su efecto encapsulante a dicha concentración, para luego decaer el porcentaje de recuperación de vitamina C a 47,15\% con 30\% de $M D$. El mismo efecto se evidenció con respecto al factor $\mathrm{D}$ y demás interacciones significativas. Además se mejora dicho efecto cuando las gotas de producto se exponen a temperaturas de entrada del aire relativamente bajas $\left(130^{\circ} \mathrm{C}\right.$ a $\left.140^{\circ} \mathrm{C}\right)$ y temperaturas de salida de $68^{\circ} \mathrm{C}$ a $71^{\circ} \mathrm{C}$, ya que las partículas se secan rápidamente formándose una capa protectora en la superficie de éstas, lo cual favorece la conservación en general de los constituyentes nutricionales del producto; sin embargo al incrementarse aún más la temperatura de entrada $\left(140^{\circ} \mathrm{C}\right.$ a $\left.150^{\circ} \mathrm{C}\right)$ y de salida $\left(71^{\circ} \mathrm{C}\right.$ a $\left.74^{\circ} \mathrm{C}\right)$ afectan significativamente la estabilidad de la vitamina $C$, disminuyendo su porcentaje de recuperación, lo cual se debe a que se caracteriza por ser una vitamina termosensible.

Efecto similar fue reportado por Hernández (2010 ) (17) quien encontró que los mayores porcentajes de recuperación de vitamina $C$ se obtenían con el incremento de la concentración de MD, velocidad de aspersión, temperatura de entrada y temperatura del aire de salida, observándose puntos de inflexión situados cerca los puntos centrales del experimento (20000 rpm, $85^{\circ} \mathrm{C}$ (factor D) y 20 - 25\% de maltodextrina), lo cual atribuyó a la disminución del efecto encapsulante de la MD por encima de dichas concentraciones, mientras que a bajas concentraciones esta no estaría en cantidad suficiente para encapsular eficientemente la vitamina $C$ de la suspensión. La vitamina $C$ y la maltodextrina se atraen debido a afinidad molecular, dado que poseen grupos $\mathrm{OH}$ en común, lo que permiten una alta interacción, ayudando a minimizar las pérdidas durante el secado por aspersión (17-19).

La concentración más baja y alta se alcanzaron en los tratamientos 5 y 7 (tabla 1) con valores de 2,703 mg/100 g (recuperación del 14,19\%) y 22,55 mg/100 g de vitamina C (recuperación del 61,13\%), respectivamente, mientras que el mayor y menor rendimiento de vitamina $C$ se obtuvo en los tratamientos 1 (2,71 mg/100 g) y $22(22,45 \mathrm{mg} / 100 \mathrm{~g})$.

El porcentaje de recuperación de vitamina $C$ en los puntos centrales fue $60,97 \% \pm 0,202 \%$, con un valor promedio de $19,218 \mathrm{mg} / 100 \mathrm{~g}$. Patil Et al. (2014) (20) reportaron consistentemente un rango de recuperación de vitamina C entre 66,7\% y $80,5 \%$ en el polvo de guayaba, lo que dependía directamente de la temperatura de entrada del aire y de la concentración de maltodextrina, los cuales al disminuir, favorecían su recuperación. Grabowski Et al. (2008) (21) al evaluar la producción de polvo de batata mediante secado por aspersión, encontró diferencias significativas en el contenido de vitamina C, las cuales atribuyó a cambios moleculares provocados por el secado. Naddaf Et al. (2012) (22) reportaron un porcentaje de recuperación de vitamina $C$ a partir del jugo de naranja secado mediante aspersión del 51,66\%, cuyo valor atribuye a la degradación de ésta durante el tratamiento térmico. Valores congruentes fueron reportados por Hernández (2010) (17) y Carrillo-Navas Et al. (2011) (23).

\section{Solubilidad}

Este parámetro presentó una diferencia significativa $(p>0,05)$ con respecto a los factores A, B, A2, B2, D2 y BD. La solubilidad del producto aumentó en la medida que se incrementaba la concentración de maltodextrina $(A)$ y la temperatura de entrada del aire caliente (B) (figura 1), lo que puede deberse a la alta solubilidad de la $M D$, que al estar en mayor concentración favorece la solubilidad del producto obtenido; este oligosacárido es de alta solubilidad, por su gran número de grupos $\mathrm{OH}$ los cuales le permiten formar puentes de hidrógeno con las moléculas de agua, hidratándose y formando moléculas esféricas al mezclarse (24).

Con respecto al factor $B$, una elevada temperatura de entrada del aire $\left(150^{\circ} \mathrm{C}\right)$ propició la pérdida de humedad, dándose por ende la rápida formación de la capa externa de las partículas. Mayores temperaturas en la superficie de la partícula incrementan la tasa evaporativa, reduciendo la humedad, quedando los puntos activos (proteínas, maltodextrina u otros) de la superficie más expuestos para la solvatación, pudiendo mejorarse así la solubilidad. Además el rango de temperaturas de salida $\left(65^{\circ} \mathrm{C}-77^{\circ} \mathrm{C}\right)$, no exhibió un efecto significativo sobre la solubilidad del producto. Estos resultados son congruentes con los reportados por Anandharamakrishnan Et al. (2008) (25) quien encontró que las proteínas del lactosuero no son afectadas significativamente a bajas temperaturas de salida $\left(60^{\circ} \mathrm{C}-\right.$ $80^{\circ} \mathrm{C}$ ) y $161^{\circ} \mathrm{C}-180^{\circ} \mathrm{C}$ en las temperaturas de entrada, durante el estudio de pérdida de solubilidad de éstas mediante secado por aspersión, rango que concuerda con las temperaturas usadas en la presente investigación; mientras que temperaturas de salida de $100^{\circ} \mathrm{C}$ a $120^{\circ} \mathrm{C}$, combinado con temperaturas de entradas de $202^{\circ} \mathrm{C}$ a $252^{\circ} \mathrm{C}$, afectan fuertemente la solubilidad de las proteínas del lactosuero, presentando la mayor pérdida

\section{TABLA 2}

Cuadrado medio del análisis de varianza del porcentaje de recuperación de la vitamina C.

\begin{tabular}{ccccccccc}
\hline F. V. & GL & $\begin{array}{c}\text { Recuperación } \\
\text { vitamina C }\end{array}$ & F. V. & GL & $\begin{array}{c}\text { Recuperación } \\
\text { vitamina C }\end{array}$ & $\begin{array}{c}\text { F. V. } \\
\text { Recuperación } \\
\text { vitamina C }\end{array}$ \\
A & 1 & $99,07^{*}$ & $B^{2}$ & 1 & $3781,69^{*}$ & AD & 1 & $31,37^{*}$ \\
B & 1 & 10,14 & $C^{2}$ & 1 & $747,20^{*}$ & BC & 1 & $4,2 \times 10-3$ \\
C & 1 & 2,63 & $D^{2}$ & 1 & $805,67^{*}$ & BD & 1 & 5,32 \\
D & 1 & $47,97^{*}$ & AB & 1 & $58,30^{*}$ & CD & 1 & 0,028 \\
A $^{2}$ & 1 & $2806,9^{*}$ & AC & 1 & 0,12 & Error & 5 & 0,0041 \\
\hline
\end{tabular}

A: Maltodextrina. B: Temperatura de entrada. C: Velocidad de aspersión. D: Temperatura de salida.

F.V., fuente de variación. G.L., grados de libertad. ${ }^{*}$, diferencia significativa $(p<0,05)$. 
de solubilidad la $\beta$-Lactoglobulina $(45,1 \%)$ en comparación con la $\alpha$-Lactoalbúmina (22,8\%). Son muchos los factores que afectan la solubilidad, tales como las condiciones de procesamiento, la composición, $\mathrm{pH}$, densidad y tamaño de partícula; sin embargo un aumento no controlado de la temperatura de proceso puede provocar el aumento en la desnaturalización de proteínas, lo que disminuye su solubilidad (26).

En la interacción BD, la solubilidad se ve favorecida en los tratamientos realizados a elevadas temperaturas de entrada $\left(150^{\circ} \mathrm{C}\right)$ y de salida $\left(74^{\circ} \mathrm{C}\right)$ alcanzando en estas condiciones la máxima solubilidad $(96,062 \%)$; por su parte la combinación de temperaturas de entrada y de salida con valores de $130^{\circ} \mathrm{C}$ (factor B) con $74^{\circ} \mathrm{C}$ (factor D) y de $150^{\circ} \mathrm{C}$ (factor B) con $68^{\circ} \mathrm{C}$ (factor D), tienen un efecto adverso en la solubilidad, presentando a estas condiciones bajos niveles de ésta $(88,872 \%$ y $89,771 \%$, respectivamente). El promedio de la solubilidad en los puntos centrales fue 93,191\% $\pm 0,795 \%$. Hernández (2010) (17) obtuvo valores consistentes de solubilidad (97,42\%) para un producto a base uchuva secado por aspersión. Valores similares fueron reportados por Moreira Et al. (2009) (27) y Bernard Et al. (2011) (28).
Fazaeli Et al. (2012) (29) reportó que el incremento en la temperatura de entrada durante el secado por aspersión del jugo de mora negra, aumentaba su solubilidad. Sin embargo en contraposición, Sahin-Nadeem Et al. (2013) (30) y Patil Et al. (2014) (20) encontraron que la solubilidad del polvo de salvia y de guayaba aumentaba al disminuir los niveles del agente encapsulante, lo cual estos últimos atribuyeron a la presencia en menor cantidad de residuos insolubles y la formación de muy pocos grumos como resultado de la utilización en menor cantidad del agente encapsulante. Chegini y Ghobadian (2005) (31) y Quek Et al. (2007) (32) atribuyeron tal efecto a la formación de una capa dura sobre la superficie de las partículas de polvo, lo que podría evitar la difusión de las moléculas de agua a través de las partículas, disminuyendo en consecuencia la humectabilidad de la partícula y la disolución del polvo.

\section{Higroscopicidad}

La variación en la higroscopicidad por efecto de los tratamientos realizados se observa en la figura 1, la cual presentó una diferencia significativa $(p<0,05)$ con respecto al factor $A$, las interacciones A2, B2 y BD. Este parámetro estuvo fuer-

\section{FIGURA 2}

Luminosidad, cromaticidad $\mathrm{a}^{*} \mathrm{y} \mathrm{b}^{*}$ en el producto en polvo obtenido.
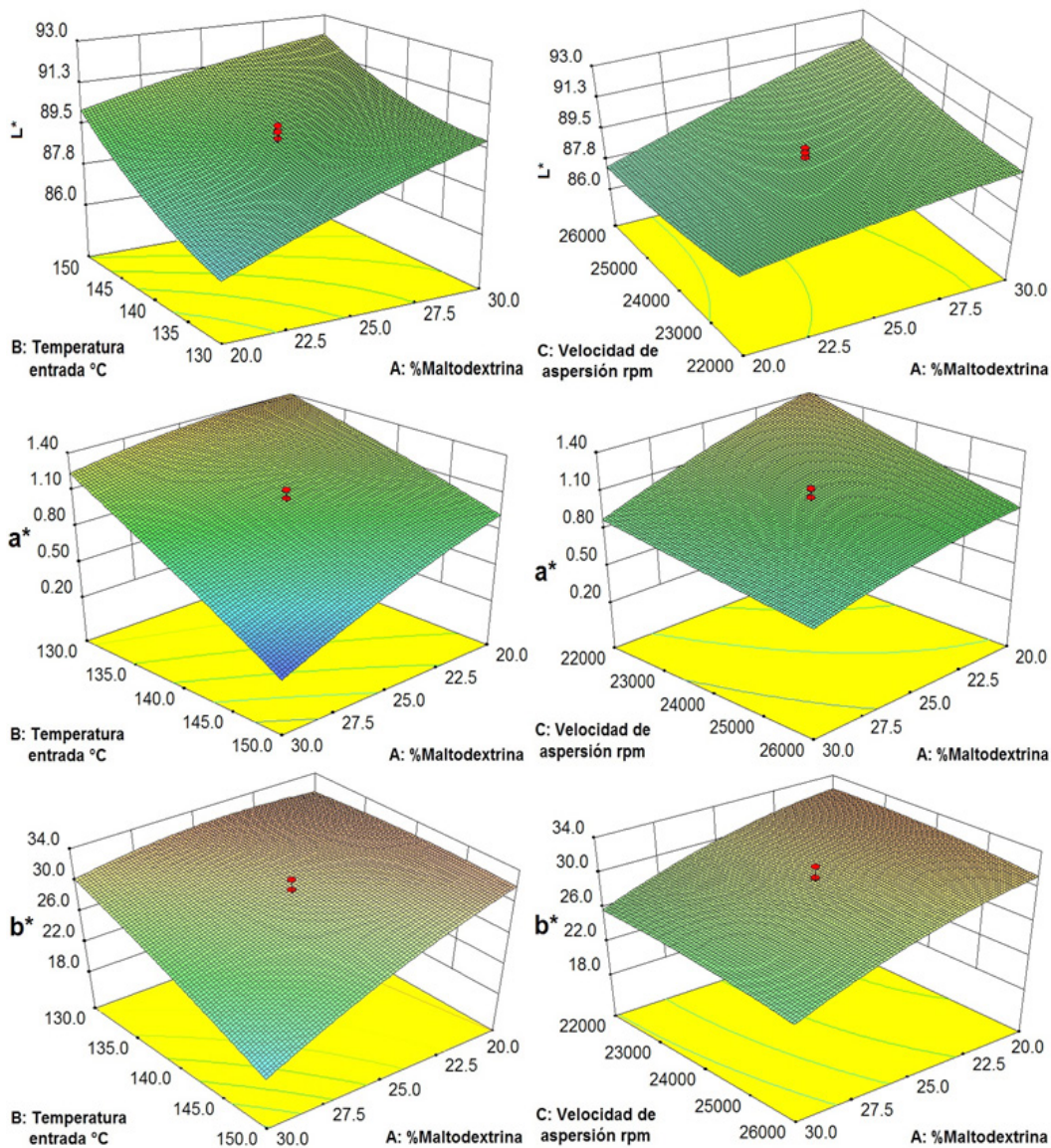
temente influenciado por el factor $A$, observándose que en la medida que aumenta la concentración de maltodextrina en la formulación, disminuye el valor de la higroscopicidad, alcanzando elevados valores $(16,830 \%)$ con $20 \%$ del factor $\mathrm{A}$ y bajos niveles a una concentración de $30 \%$ de maltodextrina $(11,575 \%)$, lo cual favorece las propiedades del producto ya que a menor higroscopicidad, menor capacidad de absorción de humedad, ayudando a su conservación. Et al. (2014) (33) también encontró que la higroscopicidad del polvo de lulo disminuía con el aumento en la concentración tanto de maltodextrina como de goma arábiga, lo cual podría estar relacionado a la naturaleza menos higroscópica de ambos componentes. Resultados similares fueron reportados por Tonon Et al. (2008) (34) y Moreira Et al. (2009) (27).

La interacción temperatura de entrada - temperatura de salida exhibió que en la medida que aumentaban estas dos variables, disminuía la higroscopicidad del producto obtenido, alcanzando un valor de $11,93 \%$ para el tratamiento a $150^{\circ} \mathrm{C}$ de temperatura de entrada y $74^{\circ} \mathrm{C}$ en la temperatura de salida. Mishra Et al. (2014) (35) reportaron que el aumento en la concentración de maltodextrina y la temperatura de entrada, disminuían de manera significativa la higroscopicidad del polvo de amla Este mismo comportamiento fue reportado por Igual Et al. (2014) (33). En contraposición Tonon Et al. (2008) (34) encontraron que los valores más bajos en la higroscopicidad del producto se obtenían en la medida que se disminuían las temperaturas del proceso; Mishra Et al. (2014) (35) atribuyó el aumento en la higroscopicidad del producto obtenido a altas temperaturas, a un elevado contenido en carbohidratos en la fruta de amla.

\section{Evaluación del color}

En la figura 2 se observa el resultado de los parámetros $L^{*}, a^{*}$ y $b^{*}$ en función de los factores de estudio. La luminosidad $\left(L^{*}\right)$ de la muestra presentó una diferencia significativa $(p<0,05)$ para los factores $A, B, D 2$ y la interacción AD. En la medida que aumenta la concentración de maltodextrina (A) y la temperatura de entrada del aire (B), la luminosidad del producto aumenta, variando de 90,0 a 92,15 a concentraciones de $20 \%$ - $30 \%$ de maltodextrina y temperatura de $150^{\circ} \mathrm{C}$. Efecto similar se presenta a una temperatura de $130^{\circ} \mathrm{C}$. La maltodextrina es un polímero de color blanco, por lo que al estar en mayor concentración en el producto aumenta el valor de $L^{*}$. El aumento en la temperatura de entrada (B) disminuye la humedad del producto lo que se podría correlacionar igualmente con el aumento en el valor de $L^{*}$, ya que al disminuir la humedad se refleja mejor el espectro de la luz que incide sobre la superficie de las partículas.

Caliskan y Dirim (2013) (36) reportaron que el aumento tanto en la concentración de maltodextrina, temperatura de entrada y salida del proceso incrementaron la luminosidad $\left(L^{*}\right)$ del polvo de zumaque. Peng Et al. (2013) (37) encontraron que la luminosidad del polvo de batata se incrementa significativamente con el aumento en la concentración de maltodextrina durante el proceso de secado por atomización, Mishra Et al. (2014) (35) al analizar el polvo de amla obtenido mediante secado por atomización, encontraron que su luminosidad se veía significativamente afectada, aumentando para una misma concentración de maltodextrina, en la medida que se incrementaba la temperatura de entrada del proceso. Resultados consistentes fueron reportados por Ahmed Et al. (2010) (38) y Jiménez-Aguilar Et al. (2011) (39).

La cromaticidad verde (-) roja (+) presentó diferencia significativa $(p<0,05)$ para los factores $A, B$ y $C$. En la medida que aumentó la concentración de maltodextrina $(A)$, la temperatura de entrada del aire (B) y velocidad de aspersión, disminuyó el valor del parámetro $\mathrm{a}^{*}$. El aumento en la concentración de maltodextrina disminuye la proporción de sólidos aportados por el mango en el producto, lo que explicaría la disminución de la tonalidad roja $\left(\mathrm{a}^{*}\right)$ al reducir los niveles de pigmentos aportados por éste; mientras que el aumento de la temperatura del aire de entrada (B) podría además afectar dichos pigmentos, lo que explicaría la disminución de $\mathrm{a}^{*}$ en la medida que aumenta el factor $B$. Independiente a las diferencias estadísticas, los cambios observados en $a^{*}$ son mínimos $(0,21$ a 1,67$)$, por lo que no se considera crítica estas variaciones. El promedio de los puntos centrales para el parámetro $a^{*}$ fue $1,022 \pm 0,05$. Peng Et al. (2013) reportaron que los valores de $\mathrm{a}^{*}$ y $\mathrm{C}^{*}$ eran significativamente más bajos con la adición del agente encapsulante, que sin éste. Quek Et al. (2007) evidenció que la adición de maltodextrina en un 10\% al jugo de sandía, disminuía el atractivo color rojo - naranja del producto en polvo obtenido por aspersión.

La cromaticidad amarilla (+) azul (-) del producto $\left(b^{*}\right)$ exhibió una diferencia significativa $(p<0,05)$ con respecto a los factores A, B y D. El valor del parámetro b* disminuyó en la medida que aumentaba la concentración de maltodextrina (A), la temperatura de entrada del aire (B) y la temperatura de salida (D). Este comportamiento se puede deber a una disminución en proporción de pulpa de mango al aumentar la concentración de maltodextrina, lo que conlleva a una disminución en la cantidad de pigmentos (carotenos) presentes en

TABLA 3

Características fisicoquímicas del producto antes y después del secado por aspersión.

\begin{tabular}{|c|c|c|c|c|}
\hline Característica & Cantidad & Recuperacion (\%) & Característica & Cantidad \\
\hline Proteína ${ }^{a}$ & $2,56 \pm 0,09$ & $86,95 \pm 0,02$ & Higroscopicidad (\%) & $94,72 \pm 0,35$ \\
\hline Grasa $^{a}$ & $1,17 \pm 0,08$ & $92,16 \pm 0,37$ & Solubilidad (\%) & $93,05 \pm 0,29$ \\
\hline Cenizas $^{a}$ & $1,24 \pm 0,11$ & $85,01 \pm 0,72$ & Solidos totales (\%) & $96,4 \pm 1,30$ \\
\hline Lactosa $^{\mathrm{a}}$ & $5,01 \pm 0,44$ & $90,03 \pm 0,62$ & $L^{*}$ & $89,55 \pm 0,02$ \\
\hline Fibra $^{a}$ & $1,40 \pm 0,15$ & $82,27 \pm 0,92$ & $a^{*}$ & $0,61 \pm 0,005$ \\
\hline Vitamina $C^{b}$ & $15,60 \pm 0,65$ & $58,15 \pm 1,27$ & $b^{*}$ & $29,43 \pm 0,01$ \\
\hline Acidez (\% p/v) & $0,191 \pm 0,02$ & ------ & Rendimiento (\%) & $65,03 \pm 1,57$ \\
\hline
\end{tabular}


el producto. Al aumentar el factor A de $20 \%$ a $30 \%$, el valor de $b^{*}$ varió de 32,22 a 25,42 mientras que al incrementar el factor $\mathrm{B}$, de $130^{\circ} \mathrm{C}$ a $150^{\circ} \mathrm{C}$, y el factor $\mathrm{D}$ de $68^{\circ} \mathrm{C}$ a $74^{\circ} \mathrm{C}$, el valor de $b^{*}$ disminuyó de 32,02 a 26,96 y 31,57 a 26,21 respectivamente. Las altas temperaturas de entrada $\left(150^{\circ} \mathrm{C}\right)$ y de salida $\left(74^{\circ} \mathrm{C}\right)$ pueden tener efecto degradativo sobre los pigmentos, razón por la cual disminuye el valor de b*. La estructura química de los carotenoides es muy susceptible a la destrucción por el calor y a la oxidación; además, puede experimentar una isomerización durante el procesamiento térmico (40). Grabowski Et al. (2008) (21) reportaron similarmente un descenso significativo en el contenido de beta-carotenos en el polvo de batata obtenido mediante secado por aspersión, causado por la isomerización de la molécula. Los carotenoides son fácilmente isomerizados por el calor, los ácidos y la luz. En general, los carotenoides presentan una configuración trans y cuando son sometidos al calentamiento, la molécula puede transformar a su configuración cis, típicamente en la posición 9,13 y 15 de los átomos de carbono. Además, los procesos de deshidratación pueden contribuir a la degradación oxidativa debido a la exposición al oxígeno y un aumento en la relación masa: superficie (41), aclarando, que dependiendo del tratamiento térmico, la configuración trans de los beta-carotenos es más propensa a la isomerización que a la degradación (42). Resultados consistentes son reportados por Candela-Cadillo Et al. (2005) (43) y Solval Et al. (2012) (44).

\section{Mejores condiciones de secado}

Al analizar los datos empleando el software Desing expert 8.0.6 nos arrojó que una concentración de maltodextrina del $27,5 \%$, temperaturas de entrada y salida de $138,2^{\circ} \mathrm{C}$ y $69,6^{\circ} \mathrm{C}$, empleando una velocidad de aspersión de $24908 \mathrm{rpm}$ se obtendrían la máxima recuperación de vitamina $C$, solubilidad, conservación del color y menor higroscopicidad en el producto, por lo que se realizó el proceso de secado a dichas condiciones obteniendo los resultados que se muestran en la tabla 3.

Todo procesamiento térmico implica la pérdida de parte de los constituyentes de un alimento, sin embargo como se observa en la tabla 3, se puede corroborar que estas se redujeron dentro de niveles aceptables, al tomar como indicador del proceso la degradación de la vitamina $C$ en el producto, por ser uno de los constituyentes más termosensibles. Se obtuvo una recuperación del porcentaje de proteína del 86,95\% mientras que la máxima cantidad de vitamina $C$ recuperada fue del $58,15 \%$.

\section{CONCLUSIÓN}

El porcentaje de recuperación de vitamina $C$ en el producto estuvo influenciada por la concentración de maltodextrina (factor A), temperatura de salida (factor D) y las interacciones $A D$ y $A B$ (maltodextrina - temperatura de entrada del aire caliente), obteniéndose la máxima recuperación en los puntos centrales de los tratamientos realizados. Al emplear el ácido ascórbico como indicador de degradación térmica durante el proceso de secado se logró maximizar la conservación de los demás constituyentes del producto como el porcentaje de proteína, grasa, fibra y lactosa cuya recuperación fue superior al $80 \%$.

\section{RESUMEN}

El mango de hilacha y el lactosuero son dos productos perecederos con gran cantidad de nutrientes. El objetivo de esta investigación fue evaluar las condiciones de secado por aspersión de un producto a base de estas materias primas tratando de obtener la máxima recuperación de vitamina $C$. El producto se secó a temperaturas de entrada del aire caliente entre $120^{\circ} \mathrm{C}-160^{\circ} \mathrm{C}$ y salida de $65^{\circ} \mathrm{C}$ a $74^{\circ} \mathrm{C}$, empleando maltodextrina entre un 15\% - 35\% como agente encapsulante. Se evaluó la concentración en vitamina C (AOAC 967.21/90) y otros parámetros como higroscopicidad, solubilidad y color del producto. Las mejores condiciones de secado se definieron utilizando una concentración de maltodextrina del 27,5\%, temperaturas de entrada y salida del aire de $138,2^{\circ} \mathrm{C}$ y $69,6^{\circ} \mathrm{C}$, con una velocidad de aspersión de $24908 \mathrm{rpm}$, obteniendo una recuperación de vitamina $C$ de 15,60 mg/100 g de producto $(58,15 \%)$ y por encima del $80 \%$ de los demás constituyentes estudiados.

Palabras clave: ácido ascórbico, maltodextrina, secado por aspersión.

Agradecimientos: A la Universidad de Córdoba por su apoyo en el desarrollo del proyecto.

\section{BIBLIOGRAFÍA}

1. Mónico P. A, Martín O, De Portela ML, Langini SH, Weisstaub AR, Greco $C$, et al. Acceptability and nutritional quality of a beverage based on orange juice and whey powder, preserved by heat or high-intensity pulsed electric fields (HIPEF). Arch Latinoam Nutr. 2006;56(4):356-60.

2. Londoño U. MM, Sepúlveda V. JU, Hernández A, Parra S. JE. Fermented fresh cheese milkwhey beverage inoculated with Lactobacillus casei. Rev Fac Nal Agr Medellin. 2008;61(1):4409-21.

3. Cuellas A, Wagner J. Elaboration of energy drink from cheese whey. Rev Lab Técnologico Uruguay. 2010;5:54-7.

4. González Siso MI. The biotechnological utilization of cheese whey: A review. Bioresour Technol. 1996;57(1):1-11.

5. Uribe JF, Estrada M, Córdoba S, Hernandez LE, Bedoya DM. Evaluation of Effective Microorganisms (EM) in production of organic fertilizer from manure bird cage. Rev Colomb Ciencias Pecu. 2001;14(2):164-72.

6. Sánchez S. GL, Gil G. MJ, Gil G. MA, Giraldo R. FJ, Millán C. LJ, Villada R. ME. Milk serum use in a company from the Antioquian Northern region by the use of efficient micro organisms. Prod Mas Limpia. 2009;4(2):65-74.

7. Baró L, Jiménez J, Martínez-Férez A, Boza J. Bioactive milk peptidos and proteins. Ars Pharm. 2001;42(3-4):135-45.

8. Ha E, Zemel MB. Functional properties of whey, whey components, and essential amino acids: Mechanisms underlying health benefits for active people (Review). J Nutr Biochem. 2003;14(5):251-8.

9. Ibrahim F, Babiker E, Yousif N, Eltinay A. Effect of fermentation on biochemical and sensory characteristics of sorghum flour supplemented with whey protein. Food Chem. 2005 Sep;92(2):285-92.

10. Parra R. Food Microencapsulation: A Review. Rev Fac Nal Agr Medellín. 2011;63(2):5669-84.

11. Ocampo A. Kinetic model of mango pulp drying. Eia. 2006;5:119-28.

12. Ribeiro SMR, Schieber A. Bioactive Compounds in Mango (Mangifera indica L.). Bioactive Foods in Promoting Health [Internet]. Ed. Academ. London: Elsevier; 2010. p. 507-23. Available from: http://linkinghub.elsevier.com/retrieve/pii/ B9780123746283000347

13. Oro JRDX, Donnamaría MC. Pharmacological action, biophysical-chemistry and dynamical structure of vitamin C. Acta Farm Bonaer. 2006;25(1):145-54.

14. Eastman JE, Moore CO. Cold water soluble granular starch 
for gelled food composition. Patent 4465702., 1984.

15. Cano-Chauca $M$, Stringheta PC, Ramos a. M, Cal-Vidal J. Effect of the carriers on the microstructure of mango powder obtained by spray drying and its functional characterization. Innov Food Sci Emerg Technol. 2005;6(4):420-8.

16. Cai YZ, Corke H. Production and Properties of Spray-dried Amaranthus Betacyanin Pigments. J Food Sci [Internet]. 2000 Oct;65(7):1248-52. Available from: http://doi.wiley. com/10.1111/j.1365-2621.2000.tb10273.x

17. Hernández G. Product development cape gooseberry (Physalis peruviana L.) Spray-dried powder added vitamin $C$ folic acid, iron and soluble fiber. Universidad Nacional de Colombia. Bogotá; 2010.

18. Dib T. CM, De Menezes HC, Santos AB, Grosso CR. Effects of drying temperature and the percentage of encapsulant on the yield Camu Camu-juice (Myrciaria dubia) microencapsulated by spray drying. 2000. p. 381-3.

19. Did T. CM, De Menezes HC, Santos AB, Grosso CRF. Study of the microencapsulation of camu-camu (Myrciaria dubia) juice. J Microencapsul. 2003;20(4):443-8.

20. Patil V, Chauhan AK, Singh RP. Optimization of the spray-drying process for developing guava powder using response surface methodology. Powder Technol [Internet]. Elsevier B.V.; 2014;253:230-6. Available from: http:// dx.doi.org/10.1016/j.powtec.2013.11.033

21. Grabowski J a., Truong VD, Daubert CR. Nutritional and rheological characterization of spray dried sweetpotato powder. LWT - Food Sci Technol. 2008;41(2):206-16.

22. Naddaf L, Avalo B, Oliveros M. Spray-dried natural orange juice encapsulants using maltodextrin and gum arabic. Rev Téc Ing Univ Zulia. 2012;35(1):20-7.

23. Carrillo-Navas H, González-Rodea DA, Cruz-Olivares J, Barrera-Pichardo JF, Román-Guerrero A, Pérez-Alonso C. Storage stability and physicochemical properties of passion Fruit juice microcapsules by spray-drying. Rev Mex Ing Quími. 2011;10(3):421-30.

24. López B, Carvajal de P. LM, Milla C. L. Establishing of the conditions of the blend of pulp with banana (Musa paradisiaca L.) to submit spray dryng. Vitae. 2009;16(3):287-96.

25. Anandharamakrishnan C, Rielly CD, Stapley a. GF. Loss of solubility of $\alpha$-lactalbumin and $\beta$-lactoglobulin during the spray drying of whey proteins. LWT - Food Sci Technol. 2008:41(2):270-7.

26. Mujumdar AS, Molnár K, Pakowski Z, Marinos-Kouris D, Maroulis ZB, Saravacos GD, et al. Handbook of Industrial Drying. CRC. 2006. 1279 p.

27. Moreira GÉG, Maia Costa MG, Souza ACR De, Brito ES De, Medeiros MDFD De, Azeredo HMC De. Physical properties of spray dried acerola pomace extract as affected by temperature and drying aids. LWT - Food Sci Technol. 2009;42(2):641-5.

28. Bernard C, Regnault S, Gendreau S, Charbonneau S, Relkin P. Enhancement of emulsifying properties of whey proteins by controlling spray-drying parameters. Food Hydrocoll [Internet]. Elsevier Ltd; 2011;25(4):758-63. Available from: http://dx.doi.org/10.1016/j.foodhyd.2010.08.011

29. Fazaeli M, Emam-Djomeh Z, Kalbasi Ashtari A, Omid M. Effect of spray drying conditions and feed composition on the physical properties of black mulberry juice powder. Food Bioprod Process [Internet]. Institution of Chemical Engineers; 2012;90(4):667-75. Available from: http:// dx.doi.org/10.1016/j.fbp.2012.04.006

30. Şahin-Nadeem H, Dinçer C, Torun M, Topuz A, Özdemir $F$. Influence of inlet air temperature and carrier mate- rial on the production of instant soluble sage (Salvia fruticosa Miller) by spray drying. LWT - Food Sci Technol. 2013;52(1):31-8.

31. Chegini G. R, Ghobadian B. Effect of spray-drying conditions on physical properties of orange juice powder. Dry Technol. 2005;23(3):657-68.

32. Quek SY, Chok NK, Swedlund P. The physicochemical properties of spray-dried watermelon powders. Chem Eng Process Process Intensif. 2007;46(5):386-92.

33. Igual M, Ramires $S$, Mosquera LH, Martínez-Navarrete $N$. Optimization of spray drying conditions for lulo (Solanum quitoense L.) pulp. Powder Technol [Internet]. Elsevier B.V.; 2014;256:233-8. Available from: http:// dx.doi.org/10.1016/j.powtec.2014.02.003

34. Tonon $R$ V., Brabet $C$, Hubinger MD. Influence of process conditions on the physicochemical properties of açai (Euterpe oleraceae Mart.) powder produced by spray drying. J Food Eng. 2008;88(3):411-8.

35. Mishra P, Mishra S, Mahanta CL. Effect of maltodextrin concentration and inlet temperature during spray drying on physicochemical and antioxidant properties of amla (Emblica officinalis) juice powder. Food Bioprod Process [Internet]. Institution of Chemical Engineers; 2014;92(3):252-8. Available from: http://dx.doi. org/10.1016/j.fbp.2013.08.003

36. Caliskan G, Nur Dirim S. The effects of the different drying conditions and the amounts of maltodextrin addition during spray drying of sumac extract. Food Bioprod Process [Internet]. Institution of Chemical Engineers; 2013;91(4):539-48. Available from: http://dx.doi. org/10.1016/j.fbp.2013.06.004

37. Peng Z, Li J, Guan Y, Zhao G. Effect of carriers on physicochemical properties, antioxidant activities and biological components of spray-dried purple sweet potato flours. LWT - Food Sci Technol [Internet]. Elsevier Ltd; 2013;51(1):348-55. Available from: http://dx.doi. org/10.1016/j.Iwt.2012.09.022

38. Ahmed M, Akter MS, Lee JC, Eun JB. Encapsulation by spray drying of bioactive components, physicochemical and morphological properties from purple sweet potato. LWT - Food Sci Technol [Internet]. Elsevier Ltd; 2010;43(9):1307-12. Available from: http://dx.doi. org/10.1016/j.Iwt.2010.05.014

39. Jiménez-Aguilar DM, Ortega-Regules a. E, Lozada-Ramírez JD, Pérez-Pérez MCI, Vernon-Carter EJ, Welti-Chanes J. Color and chemical stability of spray-dried blueberry extract using mesquite gum as wall material. J Food Compos Anal. 2011;24(6):889-94.

40. Damodaran S, Parkin KL, Fennema OR. Fennema 's food chemistry. 4th ed. Boca Raton, FL, USA: CRC; 2008. 1158 p.

41. Von Elbe JH, Schwartz SJ. Colorants. In: Dekker M, editor. Fennema Food Chemistry. New York; 1996. p. 651-722.

42. Chandler LA, Schwartz SJ. Isomerization and losses of trans$\beta$-carotene in sweet potatoes as affected by processing treatments. J Agric Food Chem. 1988;36:129-33.

43. Candelas-Cadillo MG, Alanís-Guzmán MG, BautistaJusto M, Del Río-Olague F, García-Díaz C. Lycopene content in spray-dried tomato juice. Rev Mex Ing Qum. 2005;4:299-307.

44. Solval KM, Sundararajan S, Alfaro L, Sathivel S. Development of cantaloupe (Cucumis melo) juice powders using spray drying technology. LWT - Food Sci Technol [Internet]. Elsevier Ltd; 2012;46(1):287-93. Available from: http:// dx.doi.org/10.1016/j.Iwt.2011.09.017. 